\title{
A kind of conditional connectivity of transposition networks generated by $k$-trees *
}

\author{
Weihua Yang ${ }^{\dagger}$ \\ Department of Mathematics, Taiyuan University of Technology, Shanxi Taiyuan-030024, China
}

\begin{abstract}
For a graph $G=(V, E)$, a subset $F \subset V(G)$ is called an $R_{k}$-vertex-cut of $G$ if $G-F$ is disconnected and each vertex $u \in V(G)-F$ has at least $k$ neighbors in $G-F$. The $R_{k}$-vertex-connectivity of $G$, denoted by $\kappa^{k}(G)$, is the cardinality of the minimum $R_{k}$-vertex-cut of $G$, which is a refined measure for the fault tolerance of network $G$. In this paper, we study $\kappa^{2}$ for Cayley graphs generated by $k$-trees. Let $\operatorname{Sym}(n)$ be the symmetric group on $\{1,2, \cdots, n\}$ and $\mathcal{T}$ be a set of transpositions of $\operatorname{Sym}(n)$. Let $G(\mathcal{T})$ be the graph on $n$ vertices $\{1,2, \ldots, n\}$ such that there is an edge $i j$ in $G(\mathcal{T})$ if and only if the transposition $i j \in \mathcal{T}$. The graph $G(\mathcal{T})$ is called the transposition generating graph of $\mathcal{T}$. We denote by $\operatorname{Cay}(\operatorname{Sym}(n), \mathcal{T})$ the Cayley graph generated by $G(\mathcal{T})$. The Cayley graph $\operatorname{Cay}(\operatorname{Sym}(n), \mathcal{T})$ is denoted by $T_{k} G_{n}$ if $G(\mathcal{T})$ is a $k$-tree. We determine $\kappa^{2}\left(T_{k} G_{n}\right)$ in this work. The trees are 1-trees, and the complete graph on $n$ vertices is a $n-1$-tree. Thus, in this sense, this work is a generalization of the such results on Cayley graphs generated by transposition generating trees[26] and the complete-transposition graphs 22].
\end{abstract}

Keywords: Star graphs; Bubble sort graphs; Transposition networks; Fault-tolerance; Transposition generating trees

\section{Introduction}

The interconnection network of a communication or distributed computer system is usually modeled by a (directed) graph $G$ in which the vertices represent the switching elements or processors and communication links are represented by (directed) edges. The traditional connectivity $\kappa$ and the edge connectivity $\lambda$ of a network are two classic parameters measuring fault tolerance. The higher these parameters are, the more reliable the network is [16]. However, it always underestimates the resilience of large networks. There is a discrepancy because the occurrence of events which would disrupt a large network after a few processor or link failures is highly unlikely. Thus the disruption envisaged occurs in a worst case scenario (see [10, 17] for a detailed explanation for the shortcoming of using $\kappa(G)$ to measure the network reliability). To overcome the shortcoming, Esfahanian [10]

${ }^{*}$ The research is supported by NSFC (No.11671296), The Project-sponsored by SRF for ROCS, SEM, and Fund Program for the Scientific Activities of Selected Returned Overseas Professionals in Shanxi Province.

${ }^{\dagger}$ Corresponding author. E-mail: ywh222@163.com. 
proposed the concept of restricted connectivity which is a special case of conditional connectivity proposed by Harary [12. This concept was generalized by Latifi et al. [17] to $R_{k}$-vertex-connectivity as a measure of conditional fault tolerance of networks.

A $R_{k}$-vertex-set of a graph $G$ is a vertex subset $F \subseteq V(G)$ such that every vertex $u \in V(G)-F$ has at least $k$ neighbors in $G-F$. A $R_{g}$-vertex-cut of a connected graph $G$ is a $R_{k}$-vertex-set $F$ such that $G-F$ is disconnected. The cardinality of a minimum $R_{k}$-cut of $G$ is the $R_{k}$-vertex-connectivity of $G$, denoted by $\kappa^{k}(G)$.

Let $\Gamma$ be a group and $S$ be a subset of $\Gamma \backslash\left\{1_{\Gamma}\right\}$, where $1_{\Gamma}$ is the identity of $\Gamma$. Cayley digraph $\operatorname{Cay}(\Gamma, S)$ is the digraph with vertex set $\Gamma$ and arc set $\{(g, g \cdot s): g \in \Gamma, s \in S\}$. We say that $\operatorname{arc}(g, g \cdot s)$ has label $s$. In particular, if $S^{-1}=S$, then $\operatorname{Cay}(\Gamma, S)$ is an undirected graph, called Cayley graph.

Cayley (di) graphs have a lot of properties which are desirable in an interconnection network [13, 15]: vertex symmetry makes it possible to use the same routing protocols and communication schemes at all nodes; hierarchical structure facilitates recursive constructions; high fault tolerance implies robustness, among others. The transposition networks, Cayley graphs generated by transpositions, is the most popular family such Cayley graphs. In particular, star graphs [1] and bubble sort graphs [5] are the most famous ones, see the survey [13, 15, 18, for other kinds of Cayley graphs.

Since $\kappa(G) \leq \lambda(G) \leq \delta(G)$, a graph $G$ with $\kappa(G)=\delta(G)$ is called maximally vertex connected and a graph $\mathrm{G}$ with $\lambda(G)=\delta(G)$ is called maximally edge connected. It is well-known [20] that any vertex transitive graph is maximally edge connected (Cayley (di) graphs are always vertex transitive), and many Cayley graphs are maximally vertex connected ( such as hypercubes, star graphs, bubble sort graphs, etc.). Works on $\kappa^{1}$ of Cayley graphs generated by transposition trees can be found in [6, 7, 14]. In [21], Wan and Zhang determined $\kappa^{2}$ for star graphs, which was generalized by Cheng et al. 8 to Cayley graphs generated by transposition trees (independently by Yang [26]). Zhang et al. in [28] determined $\kappa^{2}$ for alternating group graphs, and Cheng et al. 9] generalized the results in [28] to the Cayley graphs generated by 2-trees. Recently, Yu et al. [29] determined $\kappa^{2}$ for Cayley graphs generated by unicyclic graphs. Wang et al. [22] determined $\kappa^{2}$ for Cayley graphs generated by complete transposition graphs. For the related research on the $R_{k}$-vertex connectivity for the Cayley graphs, we refer to [19, 23, 24, 25, 27].

In this work, we consider Cayley graph $\operatorname{Cay}(\operatorname{Sym}(n), \mathcal{T})$, where $\operatorname{Sym}(n)$ is the symmetric group on $\{1,2, \ldots, n\}$ and $\mathcal{T}$ is a set of transpositions of $\operatorname{Sym}(n)$. Let $G(\mathcal{T})$ be the graph on $n$ vertices $\{1,2, \ldots, n\}$ such that there is an edge $i j$ in $G(\mathcal{T})$ if and only if the transposition $i j \in \mathcal{T}$. The graph $G(\mathcal{T})$ is called the transposition generating graph of $\mathcal{T}$. For convenience, we call $\operatorname{Cay}(\operatorname{Sym}(n), \mathcal{T})$ the Cayley graph generated by $G(\mathcal{T})$. In particular, if $G(\mathcal{T})$ is a path, $\operatorname{Cay}(\operatorname{Sym}(n), \mathcal{T})$ is the bubble-sort graph, denoted by $B S_{n}$. If $G(\mathcal{T})$ is a star, $\operatorname{Cay}(\operatorname{Sym}(n), \mathcal{T})$ is the well-known star graph, denoted by $S_{n}$. If $G(\mathcal{T})$ is a tree, the $\operatorname{Cay}(\operatorname{Sym}(n), \mathcal{T})$ includes the $B S_{n}$ and $S_{n}$ as its subclasses.

A $k$-tree $T_{k, n}$ with $n$ vertices is defined recursively as follows: A set of $k+1$ mutually adjacent vertices constitutes a $k$-tree $T_{k, k+1}$ and a $k$-tree $T_{k, n+1}$ is any graph obtained by joining a new vertex to $k$ mutually adjacent vertices of a $k$-tree $T_{k, n}$. One can see that if $k=1$, then $T_{k, n}$ is a tree. Thus, the $k$-trees are a generalization of trees, such a generalization of trees has been studied extensively, first in [2, 3]. If $G(\mathcal{T})$ is a $k$-tree, 
$\operatorname{Cay}(\operatorname{Sym}(n), \mathcal{T})$ is denoted by $T_{k} G_{n}$. In particular, if $k=n-1$, then $T_{n-1, n}$ is the complete graph. If $G(\mathcal{T})$ is complete, $\operatorname{Cay}(\operatorname{Sym}(n), \mathcal{T})$ is called complete-transposition graphs, denoted by $C T_{n}$. Wang et al. determined $\kappa^{2}\left(C T_{n}\right)$ in [22]. In this paper, we completely determined $\kappa^{2}\left(T_{k} G_{n}\right)$, which generalizes the results on Cayley graphs generated by transposition trees and by complete transposition graphs, as transposition trees are $T_{1, n}$ and the complete transposition graphs are $T_{n-1, n}$.
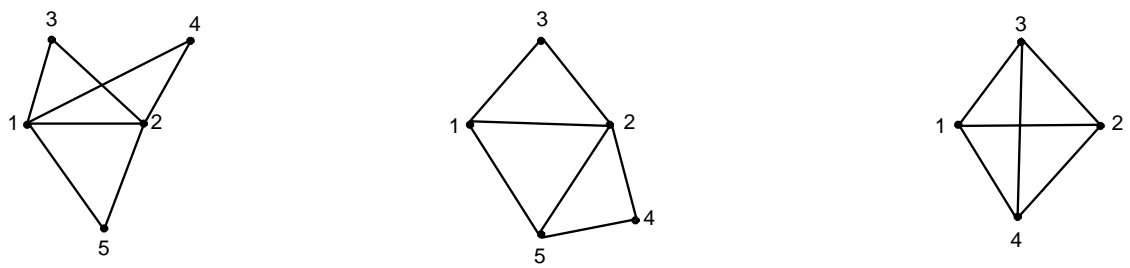

Figure 1. 2-trees on 5 vertices and 3 -tree on 4 vertices

\section{Preliminaries}

For a graph $G$, a subgraph $G_{1}$ of $G$, and a vertex $u \in V(G)$, the neighbor set of $u$ in $G_{1}$ is $N_{G_{1}}(u)=\{v$ is adjacent with $u$ in $G\}$. In particular, if $G_{1}=G$, then $N_{G}(u)$ is the neighbor set of $u$ in $G$ and $d_{G}(u)=\left|N_{G}(u)\right|$ is the degree of vertex $u$ in $G$. The minimum degree of $G$ is denoted as $\delta(G)$. For a subset $U \in V(G), N_{G}(U)=\cup_{u \in U} N_{G}(u)-U$, and $G[U]$ is the subgraph of $G$ induced by $U$. Sometimes, we use a graph itself to represent its vertex set. For example, $N_{G}\left(G_{1}\right)$ is used to denote $N_{G}\left(V\left(G_{1}\right)\right)$ where $G_{1}$ is a subgraph of $G$, and $N_{F}(U)$ is used to denote $N_{G[F]}(U)$ where $F, U$ are vertex sets. When graph $G$ is obvious in the context, we omit the subscript $G$ and use $N(U)$ to denote $N_{G}(U)$. A cycle with length $k$ is called a $k$-cycle. The length of the shortest cycle of $G$ is called the girth of $G$, denoted by $g(G)$.

In this section, we first introduce some notations and former results which will be used in our proofs. Then we shall derive some structural properties for $T_{k} G_{n}$. By the definition, a $k$-tree $T_{k, n}$ has $k n-\frac{k(k+1)}{2}$ edges and $T_{k, n}$ contains a triangle if $k \geq 2$. Thus $T_{k} G_{n}$ is a $k n-\frac{k(k+1)}{2}$ regular graph on $n$ ! vertices. For convenience, we assume $n$ is the last vertex added on $G(\mathcal{T})$, that is, the degree of $n$ in $G(\mathcal{T})$ is $k$. And we call a vertex of degree $k$ in $T_{k, n}$ is a leaf. Moreover, we assume $k \geq 2$, as the case for $k=1$ has been considered in [8, 26]. 

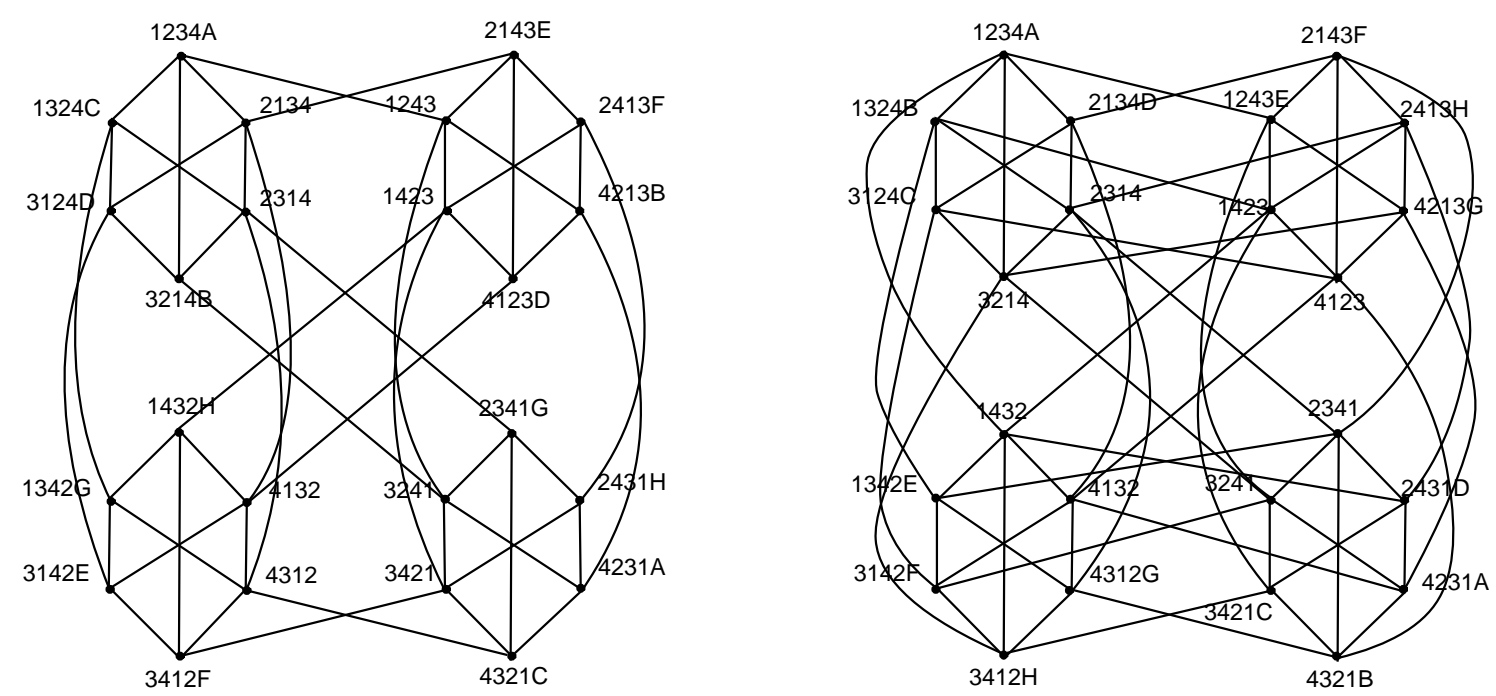

Figure 2. Cayley graphs $T_{2} G_{4}$ and $T_{3} G_{4}$.

One most important property of transposition networks is the hierarchical structure, which is particularly useful in our inductive arguments.

Assume $k<n-1$ and $n$ is a leaf of $G(\mathcal{T})$. One can see that $T_{k} G_{n}$ can be decomposed into $n$ interconnected copies of $T_{k} G_{n-1}$, say $T_{k} G_{n-1}^{1}, T_{k} G_{n-1}^{2}, \cdots, T_{k} G_{n-1}^{n}$, where $T_{k} G_{n-1}^{i}$ is the subgraph induced by vertex set $\left\{\left(p_{1} p_{2} \cdots p_{n-1} i\right) \mid\left(p_{1} p_{2} \cdots p_{n-1}\right)\right.$ ranges over all permutations of $\{1,2, \cdots, n\} \backslash\{i\}$. In particular, if $k=n-1$, then $T_{k} G_{n}$ can be decomposed into $n$ interconnected copies of $T_{k-1} G_{n-1}$, say $T_{k-1} G_{n-1}^{1}, T_{k-1} G_{n-1}^{2}, \cdots, T_{k-1} G_{n-1}^{n}$. Without loss of the generality, we always decompose $T_{k} G_{n}$ into $n$ copies by using the leaf $n$. For a vertex $u \in V\left(T_{k} G_{n-1}^{i}\right)$, we call the neighbors out of $T_{k} G_{n-1}^{i}$ outsider neighbors. By the arguments above, one can see the following.

Lemma 2.1. Let $1 \leq i, j \leq n$ be two distinct integers. Then the following holds.

1. Any vertex $u \in V\left(T_{k} G_{n-1}^{i}\right)$ has $k$ outsider neighbors in $k$ different copies.

2. The outsider neighbors of the vertices in $T_{k} G_{n-1}^{i}$ are all different.

3. There are $k(n-2)$ ! independent edges between $T_{k} G_{n-1}^{i}$ and $T_{k} G_{n-1}^{j}$. That is, $\mid N\left(T_{k} G_{n-1}^{i}\right) \cap$ $V\left(T_{k} G_{n-1}^{j}\right) \mid=k(n-2)$ !

Proof. Suppose $u=p_{1} p_{2} \cdots p_{n-1} i \in V\left(T_{k} G_{n-1}^{i}\right)$ and let $i_{1}, i_{2}, \cdots, i_{k}$ be the $k$ neighbors of $n$ in $G(\mathcal{T})$. Then $u$ has $k$ outsider neighbors $u\left(i_{1} n\right) \in V\left(T_{k} G_{n-1}^{p_{i_{1}}}\right), u\left(i_{2} n\right) \in V\left(T_{k} G_{n-1}^{p_{i_{2}}}\right)$, $\cdots, u\left(i_{k} n\right) \in V\left(T_{k} G_{n-1}^{p_{i_{k}}}\right)$. Notice that $p_{i_{k}} \neq p_{i_{l}}$ for $i_{k}, i_{l} \in\left\{i_{1}, i_{2}, \cdots, i_{k}\right\}$. Thus, (1) holds. Since (1) holds, (2) is clearly true. For a vertex $u=p_{1} p_{2} \cdots p_{n-1} i \in V\left(T_{k} G_{n-1}^{i}\right)$, if $j \in\left\{p_{i_{1}}, \cdots, p_{i_{k}}\right\}$, then there is an outsider neighbor of $u$ in $T_{k} G_{n-1}^{j}$. Assume $p_{i_{1}}=j$, then there are $(n-2)$ ! such vertices in $T_{k} G_{n-1}^{i}$ and then there are $(n-2)$ ! such outsider neighbors in $T_{k} G_{n-1}^{j}$. So the subgraph $T_{k} G_{n-1}^{i}$ implies $k(n-2)$ ! outsider neighbor in $T_{k} G_{n-1}^{j}$. Therefore, (3) holds.

The following result is due to Cheng and Lipták [6] on the Cayley graphs generated by transpositions. 
Theorem 2.1. Let $G$ be the Cayley graph obtained from a generating graph $G(\mathcal{T})$ on $\{1,2, \cdots, n\}$ with $m$ edges, where $m \geq 7$. Suppose that $T$ is a set of vertices of $G$ such that $|T| \leq 2 m-2$ if $G(\mathcal{T})$ has no triangles and $|T| \leq 2 m-3$ if $G(\mathcal{T})$ has a triangle. Then one of the following is true:

1. $G-T$ is connected.

2. $G-T$ is disconnected with exactly two components, one of which is a singleton.

3. The transposition generating graph $G(\mathcal{T})$ has no triangles, $G-T$ is disconnected with exactly two components, one of which is $K_{2}$, and $|T|=2 m-2$.

4. The transposition generating graph $G(\mathcal{T})$ has no triangles, $G-T$ is disconnected with exactly three components, two of which are singletons, and $|T|=2 m-2$.

5. The transposition generating graph $G(\mathcal{T})$ has a triangle, $G-T$ is disconnected with exactly three components, two of which are singletons, and $|T|=2 m-3$.

Notice that a $k$-tree has $k n-\frac{k(k+1)}{2}$ edges. By using the lemma above, we have the following without proof.

Corollary 2.1. For any integer $n \geq 5,2 \leq k \leq n-1$, let $T_{k} G_{n}$ be the Cayley graphs generated by a $k$-tree. Then $\kappa^{1}\left(T_{k} G_{n}\right)=2\left(\overline{k n}-\frac{\bar{k}(k+1)}{2}\right)-2$.

The girth of $T_{k} G_{n}$ is 4 . For a 4-cycle $\left(u_{1} u_{2} u_{3} u_{4}\right)$ where $u_{2}=u_{1}(i j), u_{3}=u_{2}(k l), u 4=$ $u_{3}(i j), u_{1}=u_{4}(k l)$, and $i, j, k, l$ are all distinct. Call this form of 4-cycle as Type A 4cycle. When $G(\mathcal{T})$ contains triangles, except Type A 4-cycles, there are 4-cycles in $T_{k} G_{k}$ having the form $\left(u_{1} u_{2} u_{3} u_{4}\right)$ where $u_{2}=u_{1}(s p), u_{3}=u_{2}(p t), u_{4}=u_{3}(s p), u_{1}=u_{4}(s t)$, and spt is the unique 3 -cycle in $G(\mathcal{T})$. Call this form of 4-cycle as Type B 4-cycle.

For any two distinct vertices $u, v$ in $T_{k} G_{n},|N(u) N(v)| \leq 3$. Furthermore, if $|N(u) N(v)|=$ 3 , then the three common neighbors of $u$ and $v$ have the form $u_{1}=u(s t)=v(s p), u_{2}=$ $u(s p)=v(p t)$, and $u_{3}=u(p t)=v(s t)$, where spt is a triangle in $G(\mathcal{T})$. The Cayley graph $T_{k} G_{n}$ has an important structural property bolew.

Lemma 2.2 ([6]). The Cayley graph $T_{k} G_{n}$ contains no $K_{2,4}$ as subgraphs.

Recall that we denote the grith of a graph $G$ by $g(G)$. We list the following known results due to Wang and Zhang.

Theorem 2.2 ([21]). For any integer $n \geq 4, \kappa^{2}\left(S_{n}\right)=g(n-3)$.

Cheng and Lipták [8], and Yang and Meng [26] generalized the result above to the Cayley graphs generated by transposition generating trees.

Theorem 2.3 ([8, 26]). Let $G_{n}$ be the Cayley graph generated by transposition generating tree. Then $\kappa^{2}\left(G_{n}\right)=g(n-3)$ for $n \geq 4$.

Recently, Wang et al. 22] consider the complete transposition graphs. 
Theorem $2.4([22])$. Let $C T_{n}$ be the complete transposition graph. Then $\kappa^{2}\left(C T_{n}\right)=$ $2 n(n-1)-10$ for $n \geq 5$.

For the Cayley graph generated by unicyclic graphs $U C_{n}$ (an unicyclic graph means the graph contains exactly one cycle), Yu et al. showed the following.

Theorem 2.5 (29]). Let $U C_{n}$ be the Cayley graph generated by a unicyclic graph $G(\mathcal{T})$ and $n \geq 4$. Then $\kappa^{2}\left(U C_{n}\right)=4 n-10$ if $G(\mathcal{T})$ contains a triangle, and $\kappa^{2}\left(U C_{n}\right)=4 n-10$ if $G(\mathcal{T})$ contains no triangles.

\section{Determining $\kappa^{2}\left(T_{k} G_{n}\right)$}

In this section, we shall show that $\kappa^{2}\left(T_{k} G_{n}\right)=4\left(k n-\frac{k(k+1)}{2}\right)-10$ for $n \geq 5, k \geq 2$. Wang et al. [22] showed that $\kappa^{2}\left(T_{3} G_{4}\right)=16$, but $16 \neq 4\left(k n-\frac{k(k+1)}{2}\right)-10$. So $n \geq 5$ is necessary.

Theorem 3.1. For any integers $n \geq 5, k \geq 2, \kappa^{2}\left(T_{k} G_{n}\right)=4\left(k n-\frac{k(k+1)}{2}\right)-10$.

Proof. If $k=n-1$, then the claim holds by the main results in [22]. From now on, we assume $2 \leq k \leq n-2$.

Claim. $\kappa^{2}\left(T_{k} G_{n}\right) \leq 4\left(k n-\frac{k(k+1)}{2}\right)-10$.

Assume $i j k$ be a triangle in $\mathcal{G}(\mathcal{T})$. Then $u, v=u(i j), w=v(j k), z=u(i k)=w(i j)$ forms a type B 4-cycle in $T_{k} G_{n}$, denoted by $C$. Notice that $x=u(j k)=w(i k), y=$ $v(i k)=z(j k)$ and $T_{k} G_{n}$ does not contains the $K_{2,4}$ as subgraph. Then we have $|N(C)|=$ $4\left(k n-\frac{k(k+1)}{2}\right)-10$. We shall show that $F=N(C)$ is an $R_{2}$-vertex-cut of $T_{k} G_{n}$. So it suffices to show that every vertex in $T_{k} G_{n}-F$ has at least two neighbors. This is true for vertices in $C$. Let $t$ be a vertex in $T_{k} G_{n}-F-V(C)$. Since $T_{k} G_{n}$ is a bipartite graph and thus contains no odd cycle, we must have $N_{F}(t) \subset N(\{u, w\})$ or $N_{F}(t) \subset N(\{v, z\})$. Suppose, without loss of generality, that $N_{F}(t) \subset N(\{u, w\})$. By Lemma 2.2, $u$ and $t$ have at most 3 common neighbors, otherwise, constitute a $K_{2,4}$. Similarly, $w$ and $t$ have at most 3 common neighbors. That is, $t$ is adjacent to at most six vertices in $F$. So, if $n \geq 6$, or $n=5$ and $k=3$, then $t$ has at least $k n-\frac{k(k+1)}{2}-6 \geq 3$ neighbors in $T_{k} G_{n}-F-V(C)$. That is, $F$ is an $R_{2}$-vertex-cut of $T_{k} G_{n}$ for the cases. Suppose $n=5, k=2$. The Cayley graph $T_{k} G_{n}$ can be decomposed in 5 copies of $T_{2} G_{4}$ (see Figure 2 for the $T_{2} G_{4}$ ). We pack the 4-cycle $C$ formed by $u=1324, v=2314, w=2134, z=3124$. One can see that $F$ induces an $R_{2}$-vertex-cut of the $T_{2} G_{4}$ including the 4 -cycle (in $T_{k} G_{n}$, say $T_{2} G_{4}^{1}$ ). Then, if $t$ is in $T_{2} G_{4}^{1}$, then $t$ has at least two neighbors in $T_{k} G_{n}-F-V(C)$. By Lemma 2.1 (1), if $t \notin V\left(T_{2} G_{4}^{1}\right)$, then $t$ has at most one neighbor in $F \cap V\left(T_{2} G_{4}^{1}\right)$. And $\{u, w\}(\{v, z\})$ has four outsider neighbors. So $t$ has at most five neighbors in $N(\{u, w\})$. That is, $t$ has at least $k n-\frac{k(k+1)}{2}-5=7-5=2$ neighbors in $T_{k} G_{n}-F-V(C)$. Thus, $F=N(C)$ is an $R_{2}$-vertex-cut of $T_{k} G_{n}$ with $4\left(k n-\frac{k(k+1)}{2}\right)-10$ vertices. The claim holds.

We next show that $\kappa^{2}\left(T_{k} G_{n}\right) \geq 4\left(k n-\frac{k(k+1)}{2}\right)-10$. By contradiction, we suppose that $F$ is an $R_{2}$-vertex-cut with size no more than $4\left(k n-\frac{k(k+1)}{2}\right)-11$. Let $F_{i}=V\left(T_{k} G_{n-1}^{i}\right) \cap F$. 
Case 1. $\left|F_{i}\right| \leq 2\left(k(n-1)-\frac{k(k+1)}{2}\right)-4$ for all $i$.

Notice that $T_{k} G_{n}$ is maximally connected. Then by Lemma 2.1, $T_{k} G_{n-1}^{i}-F_{i}$ either is connected or has at most one singleton. Let $J=\left\{i: T_{k} G_{n-1}^{i}-F_{i}\right.$ has a singleton $\}$. A simple count shows that $|J| \leq 4$.

Subcase $1.1 T_{k} G_{n-1}^{i}-F_{i}$ is connected for all $i$.

By Lemma 2.1, there are $k(n-2)$ ! independent edges between $T_{k} G_{n-1}^{i}$ and $T_{k} G_{n-1}^{j}$ for any pair $\{i, j\}$. Notice that $T_{k} G_{n-1}^{i}-F_{i} \subset F_{j}$ if $T_{k} G_{n-1}^{i}-F_{i}$ is disconnected to $T_{k} G_{n-1}^{j}-F_{j}$. One can see that the inequality $k(n-2) !>2\left[2\left(k(n-1)-\frac{k(k+1)}{2}\right)-4\right]$ holds if $n \geq 6$. Thus, $T_{k} G_{n-1}^{i}-F_{i}$ is connected to $T_{k} G_{n-1}^{j}-F_{j}$ for all $j \neq i$, that is, $T_{k} G_{n}-F$ is connected if $n \geq 6$, or $n=5$ and $k=3$, a contradiction. When $n=5$ and $k=2$, the inequality becomes an equality. That is, if there exists one pair $\{i, j\}$ such that $T_{k} G_{n-1}^{i}-F_{i}$ is disconnected to $T_{k} G_{n-1}^{j}-F_{j}$, then $\left|F_{i}\right|=\left|F_{j}\right|=2\left(k(n-1)-\frac{k(k+1)}{2}\right)-4$ and $|F|-\left|F_{i}\right|-\left|F_{j}\right|=2$. Therefore, there must exist an integer $l$ such that $F_{l}=\emptyset$. Since $k(n-2) !>2\left(k(n-1)-\frac{k(k+1)}{2}\right)-4 \geq\left|F_{i}\right|$ for $n \geq 5, T_{k} G_{n-1}^{l}$ is connected to $T_{k} G_{n-1}^{j}-F_{j}$ for each $j$. Thus, $T_{k} G_{n}-F$ is connected if $n \geq 5$, a contradiction.

Subcase 1.2 There is exactly one $i$ such that $T_{k} G_{n-1}^{i}-F_{i}$ is disconnected.

Without loss of generality, we assume $T_{k} G_{n-1}^{1}-F_{1}$ is disconnected, that is, $T_{k} G_{n-1}^{1}-F_{1}$ contains exactly two components such that one of them is a singleton $v$. Since $T_{k} G_{n}$ is maximally connected, we have $\left|F_{1}\right| \geq k(n-1)-\frac{k(k+1)}{2}$. By an argument similar to that of Subcase 1.1 , one can see that $T_{k} G_{n-1}^{2}-F_{2}, \cdots, T_{k} G_{n-1}^{n}-F_{n}$ are in the same component $C$ of $T_{k} G_{n}-F$. Now we show that the large component $H$ of $T_{k} G_{n-1}^{1}-F_{1}$ is also in $C$. Notice that $T_{k} G_{n-1}^{1}-F_{1}-\{v\}$ has $k\left[(n-1) !-\left|F_{1}\right|-1\right]$ outsider neighbors. However, $\left|F-F_{1}\right|<k\left[(n-1) !-\left|F_{1}\right|-1\right]$. So $T_{k} G_{n-1}^{1}-F_{1}$ is in the large component $C$, and $\{v\}$ is either a component or in $C$. This contradicts with $F$ being an $R_{2}$-vertex-cut.

Subcase 1.3 There is exactly two integer $i$ 's such that $T_{k} G_{n-1}^{i}-F_{i}$ is disconnected.

Without loss of generality, we assume that $T_{k} G_{n-1}^{1}-F_{1}$ and $T_{k} G_{n-1}^{2}-F_{2}$ are disconnected. Let $v_{1}$ and $v_{2}$ be the singletons of $T_{k} G_{n-1}^{1}-F_{1}$ and $T_{k} G_{n-1}^{2}-F_{2}$ respectively. Thus, we have $\left|F_{1}\right| \geq k(n-1)-\frac{k(k+1)}{2}$ and $\left|F_{2}\right| \geq k(n-1)-\frac{k(k+1)}{2}$. Similarly, $T_{k} G_{n-1}^{3}-F_{3}, \cdots, T_{k} G_{n-1}^{n}-F_{n}$ are in the same component $C$ of $T_{k} G_{n}-F$. Since $T_{k} G_{n-1}^{1}-F_{1}-\left\{v_{1}\right\}\left(T_{k} G_{n-1}^{2}-F_{2}-\left\{v_{2}\right\}\right)$ has at least $k\left[(n-1) !-\left|F_{1}\right|-1\right]-k(n-2)$ ! outsider neighbors in $T_{k} G_{n}-V\left(T_{k} G_{n-1}^{2}\right)$ and $\left|F-F_{1}-F_{2}\right|<k\left[(n-1) !-\left|F_{1}\right|-1\right]-k(n-2)$ !, we have that the larger components in $T_{k} G_{n-1}^{1}-F_{1}\left(T_{k} G_{n-1}^{2}-F_{2}\right)$ is also in the same component $C$. The subgraph induced by $\left\{v_{1}, v_{2}\right\}$ has degree at most one. So this contradicts with $F$ being an $R_{2}$-vertex-cut again.

Subcase 1.4 There is exactly three integer $i$ 's such that $T_{k} G_{n-1}^{i}-F_{i}$ is disconnected.

Without loss of generality, we assume that $T_{k} G_{n-1}^{1}-F_{1}, T_{k} G_{n-1}^{2}-F_{2}$ and $T_{k} G_{n-1}^{3}-F_{3}$ are disconnected such that $v_{1}, v_{2}$ and $v_{3}$ are the corresponding singletons, respectively. Similarly, $T_{k} G_{n-1}^{4}-F_{4}, \cdots, T_{k} G_{n-1}^{n}-F_{n}$ are in the same component $C$ of $T_{k} G_{n}-F$. We show that $T_{k} G_{n-1}^{i}-F_{1}-\left\{v_{i}\right\}, i=1,2,3$ is also in $C$. Notice that $T_{k} G_{n-1}^{i}-F_{1}-$ $\left\{v_{i}\right\},(i=1,2,3)$ has at least $k\left[(n-1) !-\left|F_{1}\right|-1\right]-2 k(n-2)$ ! outsider neighbors in 
$T_{k} G_{n}-V\left(T_{k} G_{n-1}^{2} \cup T_{k} G_{n-1}^{3}\right)$ and $k\left[(n-1) !-\left|F_{1}\right|-1\right]-2 k(n-2) !>\left|F-F_{1}-F_{2}-F_{3}\right|$. So $T_{k} G_{n-1}^{i}-F_{1}-\left\{v_{i}\right\}, i=1,2,3$ is also in $C$. Clearly, the subgraph induced by $\left\{v_{1}, v_{2}, v_{3}\right\}$ contains a vertex of degree at most one, a contradiction.

Subcase 1.5 There is exactly four integer $i$ 's such that $T_{k} G_{n-1}^{i}-F_{i}$ is disconnected.

Without loss of generality, we assume that $T_{k} G_{n-1}^{1}-F_{1}, T_{k} G_{n-1}^{2}-F_{2}, T_{k} G_{n-1}^{3}-F_{3}$ and $T_{k} G_{n-1}^{4}-F_{4}$ are disconnected such that $v_{1}, v_{2}, v_{3}$ and $v_{4}$ are the corresponding singletons, respectively. Similarly, $T_{k} G_{n-1}^{i}-F_{i}-\left\{v_{i}\right\}(i=1,2,3,4)$ and $T_{k} G_{n-1}^{i}-F_{i}-\left\{v_{i}\right\}(i \geq 5)$ are in the same component of $T_{k} G_{n}-F$. That is, if $F$ is an $R_{2}$-vertex-cut, then $\left\{v_{1}, v_{2}, v_{3}, v_{4}\right\}$ forms a 4-cycle.

Without loss of generality, we suppose that $v_{1} v_{2} v_{3} v_{4} v_{1}$ is a 4-cycle and $v_{1}=p_{1} p_{2} \ldots$ $2 \cdots 3 \cdots 4 \cdots p_{n-1} 1$. Then we have $v_{2}=p_{1} p_{2} \cdots 1 \cdots 3 \cdots 4 \cdots p_{n-1} 2$ and $v_{4}=p_{1} p_{2} \cdots 2$ $\cdots 3 \cdots 1 \cdots p_{n-1} 4$. On the one hand, $v_{3}=p_{1} p_{2} \cdots 1 \cdots 2 \cdots 4 \cdots p_{n-1} 3$ since $v_{2}$ is a neighbor of $v_{3}$ by using some edge due to the leaf $n$. On the other hand, $v_{3}=p_{1} p_{2} \cdots 2$ $\cdots 4 \cdots 1 \cdots p_{n-1} 3$ since $v_{4}$ is a neighbor of $v_{3}$. This is impossible.

Case 2. $\left|F_{i}\right| \geq 2\left(k(n-1)-\frac{k(k+1)}{2}\right)-3$ for some $i$.

Let $I=\left\{i:\left|F_{i}\right| \geq 2\left(k(n-1)-\frac{k(k+1)}{2}\right)-3\right\}$. Since $|F| \leq 4\left(k n-\frac{k(k+1)}{2}\right)-11$, we have $|I| \leq 2$. For $j \notin I, T_{k} G_{n-1}^{j}-F_{j}$ contains at most one singleton $u_{j}$ (if it exists).

Subcase 2.1 $|I|=1$.

Without loss of generality, we assume $I=\{1\}$. We first assume that $C=\cup_{j \neq 1} T_{k} G_{n-1}^{j}-$ $F_{j}$ is connected. Since $F$ is an $R_{2}$-vertex-cut, there is a component $H$ of $T_{k} G_{n}-F$ in $T_{k} G_{n-1}^{1}-F_{1}$. Clearly, each vertex of $H$ has at least two neighbors in $T_{k} G_{n-1}^{1}-F_{1}$. Let $P=$ $v_{1} v_{2} v_{3} v_{4}$ be a path of $H$. Since $H$ is a component of $T_{k} G_{n}-F$, each vertex $x \in N_{T_{k} G_{n-1}^{1}}(P)$ is either in $F$ or its outsider neighbors in $F$. Thus $|F| \geq|N(P)| \geq 4\left(k n-\frac{k(k+1)}{2}\right)-10$, a contradiction.

Assume $C$ is not connected. Thus there is some $T_{k} G_{n-1}^{j}-F_{j}$ containing two component one which is a singleton. Similarly, the larger component of $T_{k} G_{n-1}^{j}-F_{j}$ for $j=2,3, \cdots$ are in the same component of $T_{k} G_{n}-F$, denoted by $C^{\prime}$. Let $U$ be set of singletons of $T_{k} G_{n-1}^{j}-F_{j}$ which are disconnected to $C^{\prime}$. Clearly, $U \neq \emptyset$ since $C$ is not connected. Notice that $F$ is an $R_{2}$-vertex-cut and $u_{j}$ has at most one outsider neighbor in $T_{k} G_{n-1}^{1}$. So $|U| \geq 2$. A simple count shows that $|U| \leq 3$ since $|F| \leq 4\left(k n-\frac{k(k+1)}{2}\right)-10$. Assume $|U|=3$ and $U=\left\{u_{2}, u_{3}, u_{4}\right\}$. Each neighbor of $u_{j}, j=2,3,4$ is either in $F$ or in $T_{k} G_{n-1}^{1}$. Then we have $|F| \geq\left|F_{1}\right|+3\left(k(n-1)-\frac{k(k+1)}{2}\right)+(k-3)+2(k-2) \geq 3\left(k(n-1)-\frac{k(k+1)}{2}\right)-$ $3+2\left(k(n-1)-\frac{k(k+1)}{2}\right)+(k-3)+2(k-2)>4\left(k n-\frac{k(k+1)}{2}\right)-11 \geq|F|$, a contradiction. So $|U|=2$.

Assume, without loss of generality, $U=\left\{u_{2}, u_{3}\right\}$. Since $F$ is an $R_{2}$-vertex-cut and $u_{j}$ has at most one neighbor in $T_{k} G_{n-1}^{1}$ for $j=2,3, u_{2}$ is a outsider neighbor of $u_{3}$. Similarly, one can see that the components in $T_{k} G_{n-1}^{1}-F_{1}$, which are disconnected to the edge $u_{2} u_{3}$, are connected to $C^{\prime}$. Let $u_{2}^{\prime}$ and $u_{3}^{\prime}$ be the outsider neighbors of $u_{2}$ and $u_{3}$ respectively. Then $u_{2}^{\prime}$ (and $u_{3}^{\prime}$ ) has $k-1$ outsider neighbors in $\cup_{i=4}^{n} T_{k} G_{n-1}^{i}$ by Lemma 2.1. We have known that the edges with label $(i n)$ due to the leaf $n$ can not induce a 4 -cycle. So the 
outsider neighbor of $u_{2}^{\prime}\left(u_{3}^{\prime}\right)$ is not the neighbor of $u_{2}$ and $u_{3}$. This implies $u_{2}^{\prime}$ and $u_{3}^{\prime}$ have $2(k-1)$ outsider neighbors in $F-F_{1}-F_{2}-F_{3}-N_{\cup_{i=4}}^{n}\left(u_{2}\right)-N_{\cup_{i=4}}^{n}\left(u_{3}\right)$. Notice that $|F|-\left|F_{1}\right|-\left|N_{T_{k} G_{n-1}^{2}}\left(u_{2}\right)\right|-\left|N_{T_{k} G_{n-1}^{3}}\left(u_{3}\right)\right|-\left|N_{\cup_{i=4}}^{n}\left(u_{2}\right)\right|-\left|N_{\cup_{i=4}}^{n}\left(u_{3}\right)\right| \geq 4\left(k n-\frac{k(k+1)}{2}\right)-$ $11-\left[2\left(k(n-1)-\frac{k(k+1)}{2}\right)-3\right]-2\left[k(n-1)-\frac{k(k+1)}{2}\right]-2(k-2)<2(k-1)$. This is impossible.

Subcase 2.2 $|I|=2$.

Without loss of generality, we assume $I=\{1,2\}$. There is at most one integer $j$ such that $T_{k} G_{n-1}^{j}-F_{j}$ is disconnected and one of its two component is the singleton $u_{j}$. Let $C=\cup_{j \neq 1,2} T_{k} G_{n-1}^{j}-F_{j}$.

First, we assume $C$ is connected. Then there is a component $H$ of $T_{k} G_{n}-F$ in $\left(T_{k} G_{n-1}^{1}-F_{1}\right) \cup\left(T_{k} G_{n-1}^{2}-F_{2}\right)$ since $F$ is an $R_{2}$-vertex-cut. Clearly, the degree of each vertex in $H$ is at least 2. Notice that $T_{k} G_{n}$ is bipartite and contains no the $K_{2,4}$ as subgraph. A simple count shows that $|N(H)|>|F|$ if $4 \leq|V(H)| \leq 6$. This is impossible. Thus we assume $|V(H)| \geq 7$. Then we may take four vertices from $H \cap T_{k} G_{n-1}^{1}$ (or $\left.H \cap T_{k} G_{n-1}^{2}\right)$, say $v_{1}, v_{2}, v_{3}, v_{4}$. The four vertices have $4 k$ outsider neighbors and at most four of them are in $T_{k} G_{n-1}^{2}$. So there are at least $4 k-4$ outsider neighbors of $v_{1}, v_{2}, v_{3}, v_{4}$ in $\cup_{j=3}^{n} T_{k} G_{n-1}^{j}$. However, $|F|-\left|F_{1}\right|-\left|F_{2}\right| \leq 4 k-5$. This implies that $H$ is connected to $C$, a contradiction.

We next assume $C$ is disconnected. Then $C$ consists of two components such that one of them is a singleton. Assume that $u$ is the singleton of $C$ and let $C^{\prime}=C-\{u\}$. Without loss of generality, we assume $u \in T_{k} G_{n-1}^{3}$. Similarly, there is a component $H$ of $T_{k} G_{n}-F$ in $\left(T_{k} G_{n-1}^{1}-F_{1}\right) \cup\left(T_{k} G_{n-1}^{2}-F_{2}\right) \cup\{u\}$. If $u$ is not in $H$, then we are done (see the argument above). Thus, $u \in V(H)$. Note $u$ has at least $k-2$ outsider neighbors in $\cup_{j=4}^{n}\left(T_{k} G_{n-1}^{j}\right)$. Similarly, one may take $v_{1}, v_{2}, v_{3}$ from $H \cap T_{k} G_{n-1}^{1}$ (or $\left.H \cap T_{k} G_{n-1}^{2}\right)$, and $v_{1}, v_{2}, v_{3}$ have at least $3(k-2)$ outsider neighbors in $\cup_{j=4}^{n}\left(T_{k} G_{n-1}^{j}\right)$. Thus, $4(k-2) \leq$ $\left|F-F_{1}-F_{2}-F_{3}\right| \leq 4 k-5-\left[k(n-1)-\frac{k(k+1)}{2}\right]$. This implies $3-\left[k(n-1)-\frac{k(k+1)}{2}\right]>0$, a contradiction.

Combining Theorem 2.2, Theorem 2.3, and Theorem 2.4, we have the following corollary.

Corollary 3.1. Let $T_{k} G_{n}$ be a Cayley graph generated by a $k$-tree $G(\mathcal{T})$ and $n \geq 5$.

(1) If $k \geq 2$, then $\kappa^{2}\left(T_{k} G_{n}\right)=4\left(k n-\frac{k(k+1)}{2}\right)-10$;

(2) If $k=1$ and $G(\mathcal{T})$ is not a star, then $\kappa^{2}\left(T_{k} G_{n}\right)=4\left(k n-\frac{k(k+1)}{2}\right)-8$;

(3) If $G(\mathcal{T})$ is a star, then $\kappa^{2}\left(T_{k} G_{n}\right)=6\left(k n-\frac{k(k+1)}{2}\right)-12$.

\section{Conclusion}

In this paper, we completely determined $\kappa^{2}\left(T_{k} G_{n}\right)$, where $T_{k} G_{n}$ is a Cayley graph generated by a $k$-tree. As $k$-tree is a generalization of tree and the complete graph is also a $n-1$-tree, the result in this paper generalizes the result in [8] by Cheng and Lipták on Cayley graphs generated by transposition trees (independently by Yang and Meng [26]), and Wang's result on the complete transposition graphs [22]. One can see that the size of 
the neighborhood of a shortest cycle of a graph usually provides an upper bound for $\kappa^{2}$ of the graphs. The proof in this paper shows that $\kappa^{2}\left(T_{k} G_{n}\right)$ reaches this upper bound. In this sense, $T_{k} G_{n}$ is optimal with respect to $\kappa^{2}$, and thus has high fault tolerance measured by $\kappa^{2}$.

Currently, there are only several results on $\kappa^{2}$ for some special Cayley graphs. The problems on determining general transposition networks is still open (Cayley graphs generated by an arbitrary set of transpositions.). Combining Theorem 2.5 and the main result in this paper, we leave a problem below.

Conjecture 1. Assume that $G(\mathcal{T})$ is connected. Let $G_{n}$ the Cayley graph generated by $G(\mathcal{T})$. Then the following holds.

(1) If $G(\mathcal{T})$ contains a triangle, then $\kappa^{2}\left(G_{n}\right)=4|E(G(\mathcal{T}))|-10$ for $n \geq 5$;

(1) If $G(\mathcal{T})$ contains no triangles, then $\kappa^{2}\left(G_{n}\right)=4|E(G(\mathcal{T}))|-8$ for $n \geq 4$.

\section{References}

[1] S.B. Akers, D. Harel, B. Krishnamurthy, The star graph: An attractive alternative to the n-cube, Proc. Int. Conf. Parallel Process. (1987) 393-400.

[2] L.W. Beineke, R.E. Pippert, The number of labeled $k$-dimensional trees, Journal of Combinatorial Theory 6 (1969) 200-205.

[3] L.W. Beineke, R.E. Pippert, Properties and characterizations of $k$-trees, Mathematika 18 (1971) 141-151.

[4] N. Chang, S. Hsieh, 2, 3-Extraconnectivities of hypercube-like networks, Journal of Computer and System Sciences 79 (2013) 669-688.

[5] Z. Chou, C. Hsu, J. Sheu, Bubble-sort star graphs: a new interconnection network, in: International Conference on Parallel and Distributed Systems, 1996, pp. 41-48.

[6] E. Cheng, L. Lipták, Fault resiliency of Cayley graphs generated by transpositions, International Journal of Foundations of Computer Science 18 (2007) 1005-1022.

[7] E. Cheng, L. Lipták, Linearly many faults in Cayley graphs generated by transposition trees, Information Sciences 177 (2007) 4877-4882.

[8] E. Cheng, L. Lipták, A kind of conditional vertex connectivity of Cayley graphs generated by transposition trees, Congressus Numerantium 199 (2009) 167-173.

[9] E. Cheng, L. Lipták, W. Yang, Z. Zhang, X. Guo, A kind of conditional vertex connectivity of Cayley graphs generated by 2-trees, Information Sciences 181 (2011) 4300-4308.

[10] A.H. Esfahanian, Generalized Measure of Fault Tolerance with Application to $N$-cube Networks, IEEE Trans. Comput. 38 (1989) 1586-1591.

[11] J. Fàbrega and M.A. Fiol, On the extraconnectivity of graphs, Discrete Mathematics, 155 (1996) 49-57.

[12] F. Harary, Conditional connectivity, Networks, 13 (1983) 346-357. 
[13] M. Heydemann, Cayley graphs and interconnection networks, in: G. Hahn, G. Sabidussi (Eds.), Graph Symmetry, Kluwer Academic Publishers., Netherlands, 1997, pp. 167-224.

[14] S.C. Hu, C.B. Yang, Fault tolerance on star graphs, Proceedings of the First Aizu International Symposium on Parallel Algorithms/Architecture Synthesis (1995) 176-182.

[15] S. Lakshmivarahan, J. Jwo, S.K. Dhall, Symmetry in interconnection networks based on Cayley graphs of permutation groups: a survey, Parallel Computing 19 (1993) 361-407.

[16] P. Lai, J. Tan, C. Chang, and L. Hsu, Conditional Diagnosability Measures for Large Multiprocessor Systems, IEEE Tans. Comput. 54 (2005) 165-175.

[17] S. Latifi, M. Hegde and M. Naraghi-Pour, Conditional connectivity measures for large multiprocessor systems, IEEE Trans. Comput. 43 (1994) 218-222.

[18] Latifi, S. and P.K. Srimani, Transposition networks as a class of fault-tolerant robust networks, IEEE Trans. Parallel Distrib. Sys. 45(2) 1996 230-238.

[19] A.D. Oh and H. Choi, Generalized Measures of Fault Tolerance in $n$-Cube Networks, IEEE Trans. Parallel Distrib. Sys. 4 (1993) 702-703.

[20] R. Tindell, Connectivity of Cayley digraphs, in: Ding-Zhu Du, D. Frank Hsu (Eds.), Combinatorial Network Theory, Kluwer Academic Publishers., Netherlands, 1996, pp. 41-64.

[21] M. Wan and Z. Zhang, A kind of conditional vertex connectivity of star graphs. Applied Mathematics Letters 22 (2009) 264-267.

[22] G. Wang, H. Shi, F. Hou, Y. Bai, Some condition vertex connectivities of completetransposition graphs, Information science 295 (2015) 536-543.

[23] J. Wu and G. Guo, Fault Tolerance Measures for $m$-Ary $n$-Dimensional Hypercubes Based on Forbidden Faulty Sets, IEEE Trans. Comput. 47 (1998) 888-893.

[24] W. Yang and J. Meng, Extraconnectivity of Hypercubes, Applied Mathematics Letters 22 (2009) 887-891.

[25] W. Yang and J. Meng, Extraconnectivity of Hypercubes (II), Australasian Journal of Combinatorics 47 (2010) 189-195.

[26] W. Yang, H.Z. Li, J.X. Meng, Conditional connectivity of Cayley graphs generated by transposition trees, Information Processing Letters 110 (2010) 1027-1030.

[27] W. Yang, J.X. Meng, Generalized measures of fault tolerance in hypercube networks, Applied Mathematics Letters 25 (2012) 1335-1339.

[28] Z. Zhang, W. Xiong, W-H. Yang, A kind of conditional fault tolerance of alternating group graphs, Information Processing Letters 110 (2010) 998-1002.

[29] X. Yu, X. Huang, Z. Zhang, A kind of conditional connectivity of Cayley graphs generated by unicyclic graphs, Information Sciences 243 (2013) 86-94. 